\title{
Emerging criticality at bifurcation points in heteroclinic dynamics
}

\author{
Maximilian Voit and Hildegard Meyer-Ortmanns (1) \\ Physics and Earth Sciences, Jacobs University Bremen, P.O. Box 750561, 28725 Bremen, Germany
}

(Received 3 August 2020; accepted 22 September 2020; published 19 October 2020)

\begin{abstract}
Heteroclinic dynamics is a suitable framework to describe transient dynamics that is characteristic for ecological as well as neural systems, in particular for cognitive processes. We consider different heteroclinic networks and zoom into the dynamics that emerges right at different bifurcation points. We identify features of criticality such as a proliferation of the dynamical repertoire and slowing down of the dynamics at the very bifurcations and in their immediate vicinity. It qualifies these bifurcation points as candidates for working points in systems which store and transfer information. We observe the emergence of a heteroclinic cycle between three manifolds that are densely covered by periodic orbits. Which periodic orbits are selected sensitively depends on the initial conditions due to hidden conservation laws. Our results add to the general observation that emergent features may be expected at borderlines, here between different dynamical regimes in heteroclinic dynamics.
\end{abstract}

DOI: 10.1103/PhysRevResearch.2.043097

\section{INTRODUCTION}

From the context of phase transitions in statistical mechanics, critical phenomena are observed at (usually isolated) critical points at which the driving parameter of a phase transition takes a particular value that separates phases with qualitatively different features. In particular, at second-order phase transitions, critical phenomena are observed that are characterized by a high sensitivity to perturbations, diverging correlation lengths, critical slowing down of the dynamics, and scale invariance of response functions at and in the vicinity of the critical point. However, such features of critical phenomena, termed criticality, are not restricted to critical points in phase transitions and need not require collective phenomena between many interacting units. They may also occur at single bifurcation points which mark the border between qualitatively different dynamical regimes in systems of small sizes.

A number of experimental and theoretical evidence suggests that criticality belongs to an important organization principle of living organisms. Biological systems may be poised at criticality [1], as operating near criticality has certain functional advantages [2]. One of the trademarks is the divergence of the response or susceptibility, which is useful for biological sensing systems. The emergence of long correlations in space and time may be exploited for coordinated behavior, and together with critical slowing down it may favor slowly decaying memories [2]. The dynamical repertoire, in

\footnotetext{
*h.ortmanns@jacobs-university.de

Published by the American Physical Society under the terms of the Creative Commons Attribution 4.0 International license. Further distribution of this work must maintain attribution to the author(s) and the published article's title, journal citation, and DOI.
}

particular, the variability of possible spatio-temporal patterns is maximal at criticality.

In general, critical regimes can be observed at borders between different realizations of order and disorder in a broader sense. According to Ref. [3], working at the edge of chaos (between an ordered and a chaotic regime) may optimize the trade-off between information storage (safe in the ordered regime) and information transmission (facilitated in the chaotic regime). With respect to neural activities, various experiments seem to indicate that the neural system utilizes critical points as working points. Actually, it seems to work at diverse borderlines: at the edge of activity propagation, synchronization, global stability, and percolation [2, and references therein]. In particular, criticality in the sense of being at the edge of synchrony allows the largest diversity of synchronization patterns and scale-free avalanches of activity as reported, for example, in Refs. [4-6].

At the edge of synchrony, both types of critical points, those at phase transitions and at individual bifurcation points, are observed in the auditory system of vertebrates. In the simplest formulation, a single hair cell as the ear's sensory receptor is well described as a Stuart-Landau oscillator that corresponds to the normal form of a Hopf bifurcation. At the very bifurcation point that separates fixed-point (damped oscillatory) from oscillatory behavior, the response-to-noise ratio is maximal and provides a very efficient selection of external frequencies along with an amplification mechanism. When many such Stuart-Landau oscillators are coupled in the cochlea of the vertebrate ear, a true phase transition emerges with a critical point that turns out to be the optimal working point of the system [7-9].

Similarly, Hopf bifurcations provide the optimal working points for the temporal dynamics of resting state fluctuations in the brain when analyzed in a whole brain neural mass model [10]. Again, the local dynamics is chosen as the normal form of a Hopf bifurcation, so the network model amounts to a 
coupled system of Stuart-Landau oscillators, here also exposed to noise. The coupling matrix is determined by the connectivity structure between brain areas, fitted to empirical functional connectivity dynamics. The control parameters are the bifurcation parameters of the individual local StuartLandau dynamics and the overall coupling strength. As in the previous example, the normal form has a supercritical Hopf bifurcation where the bifurcation parameters are zero for all nodes, so the local dynamics has a stable equilibrium when they are smaller than zero, which corresponds to a low-activity asynchronous state in the presence of noise and a stable limit cycle when they are larger than zero with synchronous oscillations. As it turns out, the optimal working point in the sense that its parameter choice fits best the characteristics of the empirical functional magnetic resonance imaging data is again at the edge between asynchronous low activity and collective oscillations if all parameters are slightly on the negative side (and for a specific choice of the global coupling strength between the Stuart-Landau units).

Moreover, at this working point, the brain activity appears maximally metastable. The metastability reflects the variability of the synchronization between different nodes, that is, the fluctuations of the states of phase configurations as a function of time. This maximal metastability is in line with the general observation that the number of metastable states may be largest at criticality [11], along with the highest signal complexity with various attractors and multistability, when the models operate near criticality [12]. The advantage of a highly diverse dynamical repertoire lies in offering the possibility of storing and processing diverse items of information. Thus metastability has been proposed to form a core dynamical description of coordinated brain and behavioral activity [13].

As an alternative to coupled oscillatory units like StuartLandau oscillators, a more formalized mathematical framework to describe metastable dynamics with pronounced dwell times in the metastable states is provided by heteroclinic dynamics [14]. In heteroclinic dynamics, the system evolves from one metastable state to the next in a reproducible and ordered way. One possible realization is via winnerless competition dynamics $[15,16]$, formulated in terms of generalized Lotka-Volterra equations that predict whole sequences of discrete metastable states. In the simplest case, these metastable states correspond to saddle equilibria of the dynamics. The saddles are connected via heteroclinic orbits. Very concretely, one manifestation would be that a population of three species switches from one state in which the first species is dominant to the next state in which the second species is dominant and the like. The heteroclinic orbits may form heteroclinic sequences, which may close toward cycles or even whole heteroclinic networks. Instead of a saddle equilibrium that is a point in phase space, saddles may be limit cycles so the heteroclinic trajectory migrates from one limit cycle to the next, or even heteroclinic cycles themselves. In this case, the heteroclinic trajectory connects different heteroclinic cycles, a case that we actually consider later. Thus, in general, saddles may have higher-dimensional unstable manifolds. Heteroclinic dynamics is intrinsically transient, but reproducible and asymptotically stable if appropriate conditions are satisfied.
In relation to cognitive dynamics, the dynamical variables represent the activities of information items. At the saddles, they amount to the temporary winners in a competitive information scenario. Heteroclinic dynamics has been shown in Ref. [17] to be sensitive to the very input and robust against perturbations at the same time. According to Ref. [17], heteroclinic dynamics may describe the binding between different information modalities in the brain [18], or chunking dynamics, which the brain uses to perform information processing of long sequences by splitting them into shorter information items [19].

Also in view of cognitive dynamics, we have constructed in Ref. [20] a hierarchical heteroclinic network that describes nested and self-similar transient dynamics. The structural hierarchy in phase space amounts to a heteroclinic cycle of heteroclinic cycles of heteroclinic cycles, $n$ times iterated. Specialized to two levels, a case which we later reconsider in this paper, this hierarchy implies a modulation of fast oscillations by slow oscillations, and when coupled on a spatial grid, this hierarchy in timescales translates into the spatial hierarchy of small spirals within the arms of larger spirals. Interpreted in the language of evolutionary game theory, this construction amounts to rock-paper-scissors (RPS) games, played both between the individual players and, simultaneously, between whole subpopulations.

The phase space of the heteroclinic networks with two hierarchy levels at individual sites of a grid is already ninedimensional. When these networks are assigned to a spatial grid (e.g., of size $64 \times 64$ or even larger), we observe a large dimensional reduction of phase space if all units are diffusively coupled and synchronize toward the motion of a single heteroclinic network. In the latter case, the motion consists of a heteroclinic cycle of three heteroclinic cycles involving three saddle-equilibria each. At each saddle equilibrium, one of the nine items (species) is dominant, respectively. Depending on the choice of a certain bifurcation parameter, the two hierarchy levels are reduced to a single one or even to none. The motion is then reduced to a single heteroclinic cycle between three saddles or, for a different parameter choice, attracted by a coexistence equilibrium with all nine species coexisting.

In these previous analyses, we have located the bifurcation points in phase space to explore the different dynamical regimes, either of a single heteroclinic network as described before, or of various smaller coupled sets of such units, see also Ref. [21]. However, we have analyzed the dynamics in the "bulk" of the different regimes (that is, for parameters not close to the bifurcation points). The results differ above and below the bifurcations and may represent the bulk of the respective regimes but leave open what kind of dynamics is observed at the very bifurcation points. Now, in view of brain dynamics, it is natural to ask whether also in heteroclinic dynamics the bifurcation points themselves may serve as suitable working points, sharing features of criticality similar to Hopf bifurcations in coupled Stuart-Landau oscillators [2,10]. This is why in this paper we zoom into the dynamics at the very bifurcation points and their immediate vicinity. Dynamics different from the ones that have been identified before in bulk regimes may be expected to emerge just at the borderline between different dynamical regimes which have not been disclosed from our previous bifurcation analyses. 
The paper is organized as follows. In Sec. II, we demonstrate features of criticality in the May-Leonard model as one of the simplest realizations of a RPS game between three species or three types of information items. Next we investigate the influence of coupling between two such cycles in Sec. III. Subsequently, in Sec. IV, we study a special bifurcation point in a hierarchical heteroclinic network with two hierarchy levels. Particularly at this bifurcation point, the two hierarchy levels collapse to a single level with a heteroclinic cycle between manifolds of periodic orbits. We also comment on spatially coupled systems of such hierarchical networks in Sec. V, followed by the conclusions in Sec. VI.

\section{A SINGLE HETEROCLINIC CYCLE}

We start with a May-Leonard system [22], one of the simplest systems that exhibits a heteroclinic cycle. It is equivalent to the Busse-Heikes [23] and the Guckenheimer-Holmes cycles [24]. As studied by May and Leonard [22] in the context of population dynamics, the system corresponds to a meanfield version of a RPS game with cyclic competition that is winnerless in the sense that the winners change in the course of time. The system is defined as

$$
\mathrm{d}_{t} x_{i}=x_{i}\left(1-x_{i}-(1+c) x_{i+1}-(1-e) x_{i+2}\right),
$$

where $x_{i} \in \mathbb{R}_{0}^{+}$either represents the population density of species $i$ or an information item $i$ in cognitive dynamics. The index $i \in \mathbb{Z}_{3}$ is cyclic (we omit the modulo operation). The equation is written in a form that the parameters $c$ and $e \in \mathbb{R}^{+}$ directly correspond to the contracting and expanding eigenvalues of the single-species saddle equilibria $\xi_{i}=\left\{x \in \mathbb{R}^{3}: x_{i}=\right.$ $\left.1 \wedge x_{j}=0 \forall j \neq i\right\}$, respectively. The saddles are connected by heteroclinic orbits that form an attractive heteroclinic cycle for $c>e$. Under this condition, the three-species coexistence equilibrium $\xi^{*}=\frac{1}{3+c-e}(1,1,1)$ is unstable.

When $c$ takes a special value $c_{0}=e$, a degenerate Hopf bifurcation happens: $\xi^{*}$ changes stability together with the heteroclinic cycle, which loses its attraction for $c<e$. The degeneracy refers to the simultaneous change in the stability of the coexistence equilibrium and the heteroclinic cycle. Note that this is a nonlocal property: At the bifurcation, the flow changes not only the vicinity of $\xi^{*}$ but everywhere between $\xi^{*}$ and the boundary of the phase space. Implications of this nonlocal property will be seen in critical slowing down, discussed below.

\section{A. Proliferation of the dynamics at the bifurcation point due to conserved quantities}

Precisely at the bifurcation point, the system develops conserved quantities as can be seen as follows. Rewriting Eq. (1) as

$$
\begin{aligned}
\mathrm{d}_{t} x_{i} & =x_{i}\left(1-\sum_{j} x_{j}\right)-x_{i}\left(c x_{i+1}-e x_{i+2}\right) \\
& =x_{i}\left(1-\sum_{j} x_{j}\right)-x_{i} \sum_{j \neq i} A_{i j} x_{j},
\end{aligned}
$$

we see that the first term forces the dynamics toward the plane $\mathcal{P}=\left\{\mathbf{x}: \sum_{i} x_{i}=1\right\}$, where the system ends up if the (a)

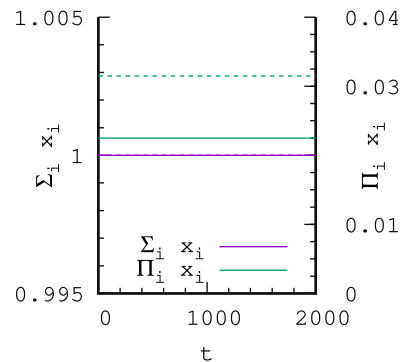

(b)

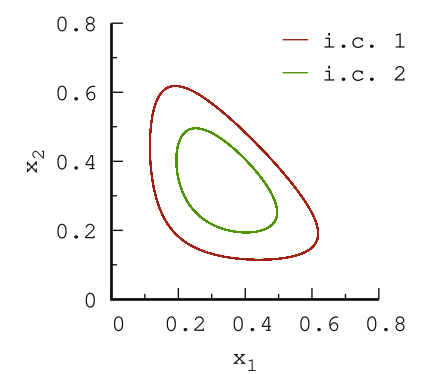

FIG. 1. (a) Dynamics of the two conserved quantities right at the bifurcation point $c=e$. Solid lines for the initial condition $(0.25,0.15,0.6)$ (i.c. 1$)$, dashed lines for $(0.35,0.2,0.45)$ (i.c. 2$)$. Note that the dashed purple line coincides with the solid purple line. (b) Two out of an infinity of periodic orbits, projected to the $x_{1}-x_{2}$ plane for the two initial conditions. Parameters are $e=0.2=c_{0}$.

second term vanishes. This is the case if the predation matrix A (with entries $A_{i, i+1}=c, A_{i, i+2}=-e$, and remaining entries 0 ) becomes skew symmetric, as happens for $c=e$ :

$$
\mathbf{A}=\left(\begin{array}{ccc}
0 & c & -e \\
-e & 0 & c \\
c & -e & 0
\end{array}\right)
$$

From extensive studies of conservative systems in population dynamics [25-27], it is known that skew symmetry of the predation matrix $\left(\mathbf{A}^{T}=-\mathbf{A}\right)$ is the essential property that guarantees conservative dynamics in general Lotka-Volterra equations of the form $\mathrm{d}_{t} x_{i}=x_{i}(\mathbf{A x})_{i}$.

If the skew symmetry refers to predation rates, such that each deletion of an individual has a counterpart of generation of an individual, (not necessarily the same one), such a process amounts to a zero-sum game as the gain and losses compensate each other. The total number of individuals is then conserved as well as the sum of concentrations $s=\sum_{i} x_{i}$ in a continuous formulation as considered here. Thus the dynamics can be restricted to an $s-1$-dimensional subspace where all concentrations are nonnegative and add up to 1 . (In our case, this is a plane in three-dimensional space.) Moreover, it was shown in Ref. [27] that within this subspace, for each linearly independent element of the kernel of the predation matrix $\mathbf{A}$, there exists one conserved quantity.

In our three-species system, the kernel of $\mathbf{A}$ is onedimensional and spanned by the vector $(1,1,1)$. Therefore, we expect one further conserved quantity once our system Eq. (2) reaches the plane $\mathcal{P}$, where it plays a zero-sum game for $c=$ $c_{0}=e$. The conserved quantity is the product of population densities $p=\Pi_{i} x_{i}, i \in\{1,2,3\}$. This result for three species is a special case of the results on conservative Lotka-Volterra networks as considered in Ref. [27].

If the initial conditions are chosen outside the plane $\mathcal{P}$, the dynamics is attracted fast toward $\mathcal{P}$, and then remains close to $\mathcal{P}$, where it is slow. For $c \neq e$ (no skew symmetric predation matrix), the dynamics is attracted to a slow (in general curved) manifold that replaces the plane in this case.

The constant values of both quantities are plotted in Fig. 1 for two initial conditions. Note that the product is constant but its value depends on the initial conditions. To each possible 
(a)

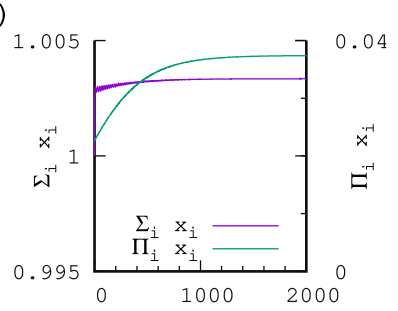

$\mathrm{t}$

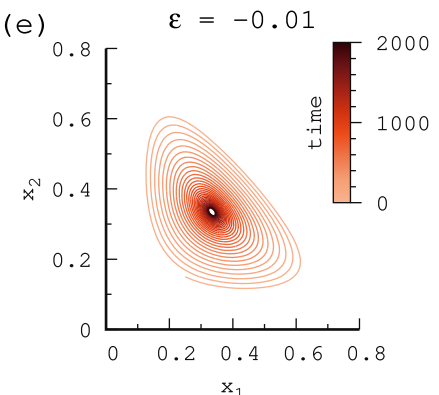

(b)
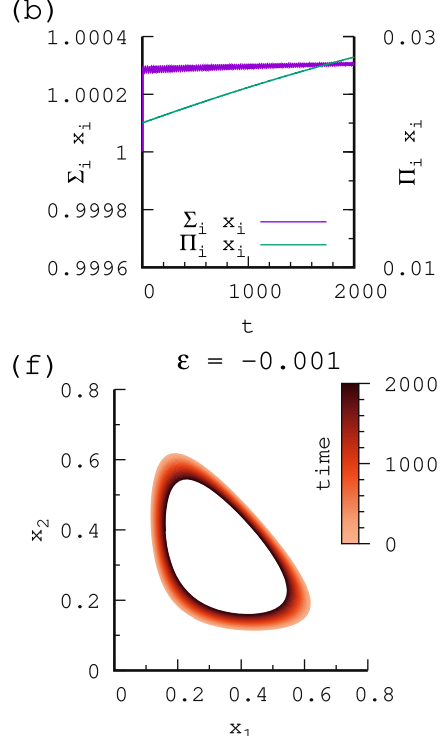

(c)

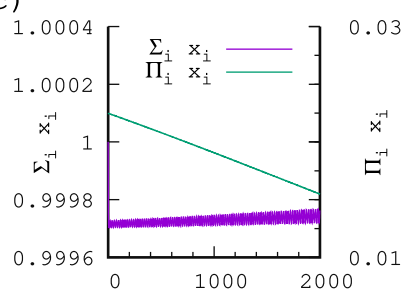

$t$

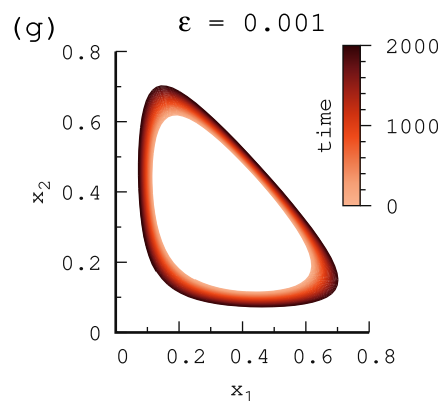

(d)

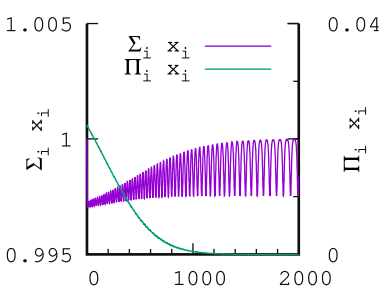

(h)

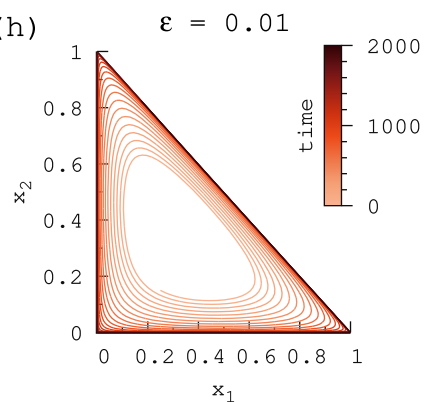

FIG. 2. Dynamics away from the critical regime (a), (d), (e), and (h) and within the critical regime (b), (c), (f), and (g) to both sides of the bifurcation point. Panels (a)-(d) show the time evolution of the quantities $\sum_{i} x_{i}$ and $\Pi_{i} x_{i}$, panels (e)-(h) display the dynamics projected to the $x_{1}-x_{2}$ plane, with color coding time. As the bifurcation occurs at $c_{0}=0.2$, panels (b) and (f) are slightly below, panels (c) and (g) slightly above the bifurcation point, on both sides still in the critical regime with scaling. For comparison, panels (a) and (e) and (d) and (h) show the situation of the critical regime, farther below and above the bifurcation point, respectively. Parameters: $e=0.2=c_{0}$. All runs started from the same initial condition: $(0.25,0.15,0.6)$.

value of $p$ belongs one periodic orbit (with specific amplitude), and all initial conditions corresponding to that specific $p$ value lead to the same periodic orbit. This means that the system strongly depends on the initial conditions as they fix the orbit. This leads to a proliferation of states, differing in the amplitudes of the periodic orbits that emerge at the bifurcation point. In contrast, the sum $\sum_{i} x_{i}$ is unity for both chosen (and any other) initial conditions [Fig. 1(a)], as the slow manifold coincides with the plane $\mathcal{P}$ precisely at the bifurcation point $c=c_{0}=e$. Figure 1(b) shows just two out of an infinity of stable periodic orbits. Note that this feature is restricted to a singular point where the condition $c=e$ is fulfilled, but it has its precursors in the vicinity of the bifurcation point as we shall see in the next section.

\section{B. The critical regime with slowing down of the dynamics and scaling}

From phase transitions, it is well known that critical behavior has characteristic precursors in the vicinity of a critical point, in the critical regime. For example, at the transition from laminar to turbulent flow in a pipe, below the transition point to turbulence though in its immediate vicinity, turbulent puffs form temporarily in the laminar flow as precursors of the new phase, but decay after some time. The decay time increases with decreasing distance to the transition point at which the turbulent phase stabilizes (see, for example, Ref. [28]). Similarly here, the proliferation of periodic orbits has precursors slightly off the bifurcation point. For $c=e+\epsilon$ with $0<|\epsilon| \ll 1$, we find dynamics that looks like a periodic orbit when viewed for short periods of time, but integrating for longer times it turns out to converge to either the coexistence equilibrium or to the heteroclinic cycle [depending on $\operatorname{sgn}(\epsilon)$ ].
This is illustrated in Fig. 2. Here, the relation $1=\sum_{i} x_{i}$ is slightly violated, since the slow manifold (on which the dynamics proceeds) does not coincide with plane $\mathcal{P}$ for $c \neq e$. While the conserved quantity is changing [Figs. 2(b) and 2(c) where these quantities don't have enough time to reach their saturation values], the periodic orbits are slowly crawling inward toward the three-species coexistence equilibrium $\xi^{*}$ [Fig. 2(f)] or slowly crawling outward toward the heteroclinic cycle [Fig. 2(g)] if we start with initial conditions far off from the final destination. Obviously 2000 time units are not enough to get at least close to their destinations.

To emphasize that we have to deal with an additional slowing down of the dynamics all over the manifold in the critical regime, we compare the dynamics inside the critical regime with that outside in Figs. 2(e) and 2(h) and display what happens over 2000 time units in all cases. As is well known, the dynamics always slows down upon reaching a heteroclinic cycle and upon approaching an equilibrium. Strictly speaking, both limits are reached only in the infinite-time limit, also here outside the critical regime, but within 2000 time units, the conserved sum and product quickly reach their final values in the case of the product in Figs. 2(a) and 2(d) and the sum in Fig. 2(a), and the bounded oscillations in case of the sum in Fig. 2(d). They approach values or oscillations, respectively, which correspond to the coexistence equilibrium Fig. 2(a) and the heteroclinic cycle Fig. 2(d).

Accordingly, starting in Fig. 2(e) from the same initial condition as in Fig. 2(f), the periodic orbit converges quickly toward the vicinity of the equilibrium and slows down only in its immediate vicinity as seen from the dense trajectories in the neighborhood of the equilibrium; similarly for the approach of the heteroclinic cycle Fig. 2(h), the trajectory first moves quickly outward and slows down only later close to 

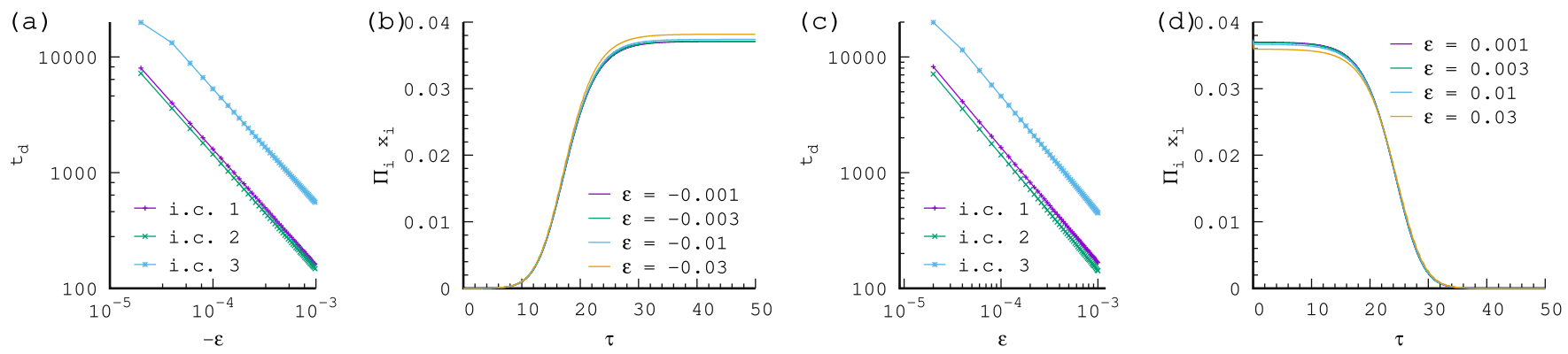

FIG. 3. Scaling of two decay times with $\epsilon$. (a) We plot the decay time $t_{d}$ against the distance from the bifurcation point $\epsilon$ double logarithmically. A periodic orbit is said to have decayed once $p$ changed by $\eta=0.0005$. Colors correspond to three different initial conditions (i.c.). (b) Decay of the product $\prod_{i=1}^{3} x_{i}$ as a function of rescaled time $\tau$ for different distances $\epsilon$ above the bifurcation point. The initial condition lies very close to one of the saddles of the heteroclinic cycle. (c) Like (a) but for positive $\epsilon$. (d) Like (b) but for positive $\epsilon$. The initial condition lies very close to the coexistence equilibrium.

the heteroclinic orbit. The additional slowing down all over the plane in the critical regime is due to the degenerate Hopf bifurcation at which also the heteroclinic cycle changes its stability, leading to a slow manifold. This manifold deserves the attribute "slow" not only because the dynamics within this manifold is slow as compared to the fast motion transverse to it, but also in the sense that the slow dynamics is not restricted to the neighborhood of the equilibrium point or the heteroclinic cycle: In addition, also away from the equilibrium point and heteroclinic cycle, the product over species concentrations slowly changes in absolute time $t$ over the intermediate time interval in Fig. 2(b), where the slope seems steep but only after rescaling time $t=\epsilon \tau$ to $\tau$.

\section{Scaling of the decay time of the periodic orbits and the product over species}

We quantify the scaling behavior of these long transient trajectories observed in the critical regime by exploiting the fact that for an actual periodic orbit, $p$ is constant and for $\epsilon \neq 0$, the value of $p$ will differ from its initial value by a small factor, here chosen as $f=0.002$, after some time $t_{d}$. Figures 3(a) and 3(c) show how the decay time $t_{d}$ of the (formerly conserved) quantity $p$ depends on the distance $\epsilon=$ $c-c_{0}$ from the bifurcation point $c_{0}=e$. The critical exponent is -1 . Different initial conditions lead to the same slope in the double-logarithmic plot, but to different prefactors, corresponding to shifts of the straight lines.

The slow decay of the periodic orbit is accompanied by the slow decay of the conserved quantity $p=\prod_{i=1} x_{i}$, for which the scaling can be easily derived as follows. The limit values of the product $\prod_{i=1} x_{i}$ are zero on the heteroclinic cycle and $1 / 27$ at the coexistence equilibrium. We determine its time evolution from eq. 1 and insert $c=e+\epsilon$ to find

$$
\mathrm{d}_{t} \prod_{i} x_{i}=\prod_{i} x_{i}\left(3\left(1-\sum_{i} x_{i}\right)-\epsilon \sum_{i} x_{i}\right) .
$$

Since the system is close to the slow manifold, we assume $\sum_{i} x_{i}=1$ (which is legitimate as for $\epsilon \rightarrow 0$ the slow manifold converges to the plane defined by that equation), which yields

$$
\mathrm{d}_{t} \prod_{i} x_{i} \approx-\epsilon \prod_{i} x_{i} \sum_{i} x_{i}
$$

Thus, by rescaling the time $t$ to $\tau=t \cdot \epsilon$, the distance from the bifurcation point is absorbed. This corresponds to collapsing $p_{\epsilon}(t)$ curves for different distances $\epsilon$ to a master curve, as shown in Figs. 3(b) and 3(d). The small vertical offset in these plots is due to the coexistence equilibrium slightly shifting with $\epsilon$ [as located at $1 /(3+c-e)(1,1,1)=$ $1 /(3+\epsilon)(1,1,1)$, so $p=1 /(3+\epsilon)^{3}$ at the coexistence equilibrium].

\section{TWO COUPLED HETEROCLINIC CYCLES}

In this section, we study the effect of coupling between two May-Leonard systems,

$$
\begin{aligned}
\mathrm{d}_{t} x_{k, i}= & x_{k, i}\left(1-x_{k, i}-(1+c) x_{k, i+1}\right. \\
& \left.-(1-e) x_{k, i+2}\right)+\delta\left(x_{k+1, i}-x_{k, i}\right),
\end{aligned}
$$

with $\delta \in \mathbb{R}^{+}$the coupling constant, the index $k \in\{1,2\}$ labeling the unit, $i \in\{1,2,3\}$ the species as before. For $c<e$, both units synchronize to the coexistence-equilibrium $\xi^{*}$, for $c>e$ they show collective slowing down, converging in unison to a synchronized heteroclinic cycle. We expect that for $c=e$ we see again a proliferation of states, in which both individual units will end up synchronizing to the same periodic orbit. Thus it is of interest of how the synchronization speed, determined by the coupling strength $\delta$, competes with the slow dynamics on the subspaces. In Fig. 4, we show two cases of small $(\delta=0.0001)$ and large $(\delta=0.01)$ couplings. If the units start from different initial conditions, they first approach different periodic orbits on the slow manifold, and then converge towards each other. As seen from a comparison between Figs. 4(a) and 4(b), the approach toward synchronized periodic orbits happens the faster the stronger the coupling. Both trajectories move inward for small coupling slowly crawling on the manifold, and synchronizing to a common periodic orbit with a small radius, smaller than both initial ones. We expect that even for very small coupling, the periodic orbits of the individual units synchronize as suggested by Figs. 4(c) and $4(\mathrm{~d})$.

Thus, again we observe a proliferation of states generated by the choice of the initial conditions. Interestingly, however, the amplitude of the periodic orbit that is finally reached depends not only on the initial conditions which fix the 

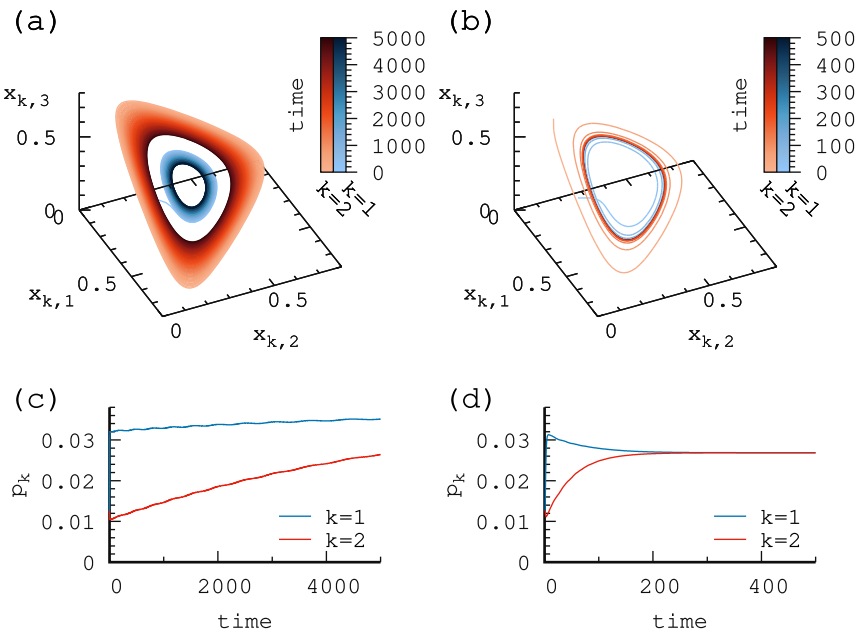

FIG. 4. Dynamics at the bifurcation point in two coupled heteroclinic cycles, exemplarily for small and large coupling strengths. The shades of colors code the time with light (dark) color for early (late) times. Trajectories of the two units, plotted in red and blue, respectively, approach each other and synchronize finally, the sooner, the stronger the coupling. (a) Weak coupling with $\delta=0.0001$. (b) Strong coupling with $\delta=0.01$. (c) and (d) display the time evolution of the products $p_{k}$ for the trajectories in (a) and (b), respectively. Initial conditions: $\mathbf{x}_{1}=(0.2,0.1,0.2)$ and $\mathbf{x}_{2}=(0.2,0.1,0.9)$. Parameters: $c=e=0.2$

conserved quantities $p_{k}=\prod_{i=1}^{3} x_{k, i}$ of both units, but also on the coupling strength.

In the critical regime, depending on the coupling strength, the units synchronize to periodic orbits or stay separate before they decay towards the coexistence equilibrium or to the heteroclinic cycle.

If we set $c=e$ only for one unit, but leave $c \neq e$ in the other, the latter one entrains the former to either converge to the heteroclinic cycle (for $c>e$ ) or to the coexistence equilibrium (for $c<e$ ). As before, this process may become very slow, when $c-e$ is small.

\section{A HETEROCLINIC CYCLE BETWEEN MANIFOLDS}

The manifold of periodic orbits found at the critical point of systems like the May-Leonard system Eq. (1) may appear embedded in a larger phase space and then lead to new effects. In this section, we study a hierarchical heteroclinic network that contains three of the manifolds found in Sec. II. Before we report the dynamics emerging at a certain bifurcation point, we first summarize previous results from Refs. [29,30] to make the paper more self-contained.

The system of generalized Lotka-Volterra equations is given as

$$
\mathrm{d}_{t} s_{i}=s_{i}-\gamma s_{i}^{2}-\sum_{j \neq i} A_{i, j} s_{i} s_{j}, \quad i \in\{1, \ldots, 9\},
$$

where $s_{i}$ denotes the population density of the $i$ th species in the language of ecological systems, or the $i$ th informational item in relation to cognitive dynamics, $\gamma$ is the death rate, the birth rate is chosen as 1 to set the timescale, and $A_{i, j}$ denotes the rate by which population $j$ preys on population $i$. The set of $A_{i, j}$ constitutes again the predation matrix $\mathbf{A}$, which here is chosen in such a way that winnerless competition results in two hierarchical levels for a certain range of parameters $[29,30]$ so fast oscillations on the short timescale are modulated by slow oscillations on the long timescale.

The hierarchy in timescales is induced by a hierarchical heteroclinic network consisting of three small heteroclinic cycles (SHCs) (each between three saddle equilibria) which are linked together via a large heteroclinic cycle (LHC) between the SHCs as its saddles. To achieve the hierarchical structure, the predation matrix $\mathbf{A}$ is chosen as the following block matrix for two hierarchy levels: Its diagonal consists of $3 \times 3$ blocks of the form $\mathbf{m}_{1}$, similar to the RPS-predation matrix for which $p^{(1)}=1$ and $o^{(1)}=0$. The off-diagonal blocks $\mathbf{p}_{2}$ have $p^{(2)}$ on their diagonal and $r$ for the remaining elements, $\mathbf{o}_{2}$ is chosen accordingly:

$$
\mathbf{A}=\left(\begin{array}{ccc}
\mathbf{m}_{1} & \mathbf{p}_{2} & \mathbf{o}_{2} \\
\mathbf{o}_{2} & \mathbf{m}_{1} & \mathbf{p}_{2} \\
\mathbf{p}_{2} & \mathbf{o}_{2} & \mathbf{m}_{1}
\end{array}\right), \mathbf{m}_{1}=\left(\begin{array}{ccc}
0 & p^{(1)} & o^{(1)} \\
o^{(1)} & 0 & p^{(1)} \\
p^{(1)} & o^{(1)} & 0
\end{array}\right) .
$$

Note the similarity of the block form of $\mathbf{A}$ to the form of $\mathbf{m}_{1}$. The independent parameters are $o^{(n)}, p^{(n)}$ and $r$ in case of $n$ hierarchy levels. To simplify the notation in the following, we identify $c=p^{(1)}, d=p^{(2)}, e=o^{(1)}, f=o^{(2)}$, and $r$ representing the remaining elements in $\mathbf{p}_{2}, \mathbf{o}_{2}$.

This number of independent parameters is dictated by the conditions on the predation rates $(0<e<f<\gamma \wedge e>2 \gamma-$ $e \wedge d>2 \gamma-e \wedge r>\gamma$ for two hierarchy levels). These very conditions are essential for making the heteroclinic network attractive, that is, guaranteeing the time evolution of the trajectory along the prescribed path with a selected heteroclinic cycle of heteroclinic cycles in the nine-dimensional phase space $[29,31]$.

The death rate $\gamma$ plays the role of a bifurcation parameter. If we tune $\gamma$ from smaller to larger values, the system undergoes a sequence of Hopf bifurcations. One type of them happens simultaneously at all three coexistence equilibria, involving three species each. When they become attractive in their subspace, the lowest level of the hierarchical heteroclinic network collapses and the remaining LHCs now connect the three-species coexistence saddle equilibria. The second type of Hopf bifurcations happens all at the global (nine-species) coexistence equilibrium. When this equilibrium becomes attractive, the second hierarchy level also collapses, corresponding to the slow timescale.

The order of bifurcations depends on the choice of the other parameters. For example, for $r=1.25, e=0.2, f=0.3$, and $c=d=2$, two hierarchy levels are observed below $\gamma=1.1$. Hopf bifurcations then occur at

$$
\gamma_{1,2}^{(g)}=1.0<\gamma^{(c)}=1.1<\gamma_{3}^{(g)}=1.3<\gamma_{4}^{(g)}=1.45,
$$

with the superscript $g$ denoting that the Hopf bifurcation proceeds at the global coexistence equilibrium with nine coexisting species (note that there are several global Hopf bifurcations as there exist multiple pairs of complex conjugated eigenvalues at this equilibrium), while the superscript $c$ refers to the bifurcation at the three-species saddle equilibria. Both, $\gamma^{(c)}$ and $\gamma_{4}^{(g)}$ lead to a qualitative change of the 
dynamics: At $\gamma=1.1$, the hierarchy is reduced from two levels to one level; the former hierarchical heteroclinic network becomes a heteroclinic cycle between three-species saddle equilibria. At $\gamma=1.45$, the hierarchy is reduced from this remaining level to zero levels, as the global coexistence equilibrium becomes stable. The first of these transitions is the one in which we are interested here, as a zoom into the dynamics at this point reveals a new invariant set: a heteroclinic cycle that approaches entire manifolds as its saddles.

\section{A. Proliferation of the dynamics at one of the bifurcation points}

Precisely at the bifurcation point $\gamma=1.1$ within each of the three subspaces,

$$
\mathcal{S}_{i}=\left\{\mathbf{s} \in \mathbb{R}_{+}^{9}: s_{j}=0 \forall j \leqslant 3(i-1) \vee j>3 i\right\} \cong \mathbb{R}_{+}^{3},
$$

we find the same situation as in Sec. II: There exists a slow manifold densely populated by periodic orbits, which coincides with the plane $\mathcal{P}_{i}=\left\{\mathbf{s}: \sum_{3(i-1) \leqslant j<3 i} x_{j}=\frac{1}{\gamma}\right\} \subset \mathcal{S}_{i}$. This is seen as follows. Let us first rewrite Eq. (7) as

$$
\mathrm{d}_{t} s_{i}=s_{i}\left(1-\sum_{j} \gamma s_{j}\right)-s_{i} \sum_{j \neq i}\left(A_{i, j}-\gamma\right) s_{j},
$$

constrained to subspace $\mathcal{S}_{i}$. When the system approaches the planes $\mathcal{P}_{i}$ which have saddle-type stability in the directions transverse to their respective subspace, the trajectory comes closer and closer to these planes in iterated revolutions of the heteroclinic trajectory associated with the LHC. The first term of Eq. (11) itself attracts the trajectory to the plane while the second term produces a zero-sum game. Let us consider the subspace $\mathcal{S}_{1}$, spanned by $s_{1}, s_{2}, s_{3}$. In this case, the second term of Eq. (11) amounts to a zero-sum game for nonvanishing $s_{1}, s_{2}, s_{3}$ and almost vanishing other species close to this plane, with an effective predation matrix,

$$
B_{i, j}=A_{i, j}-\gamma\left(1-\delta_{i, j}\right), \quad i, j \in\{1,2,3\},
$$

that is skew symmetric at the bifurcation point $\gamma=\frac{c+e}{2}$ for the first three species, while the asymmetric parts of the matrix do not contribute, because species four through nine almost vanish close to $\mathcal{S}_{1}$. Thus, as long as the trajectory is in the vicinity of this subspace, it "feels" the conservative dynamics on the plane that constitutes the slow manifold. It then temporarily "selects" one of the infinitely many periodic orbits with a certain amplitude.

A remark is in order on what we term a periodic orbit in the following. Strictly speaking, we find a periodic orbit only as long as the initial coordinates of the trajectory are assigned within one of the subspaces. In the immediate vicinity of the subspace, we still term a periodic orbit what is actually an unclosed trajectory as it spirals close to and out of the plane before and after seemingly periodic motion. In the course of time, the subspaces are approached closer and closer as they correspond to saddles of the heteroclinic cycle in (a)

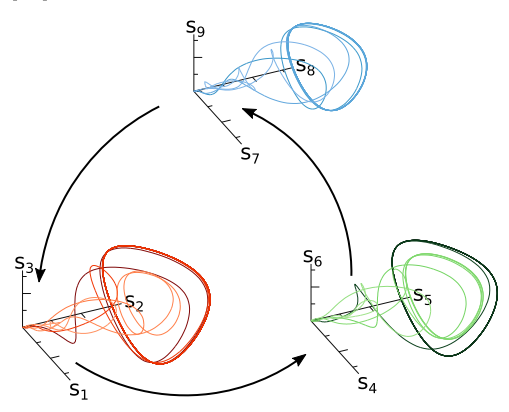

(d)

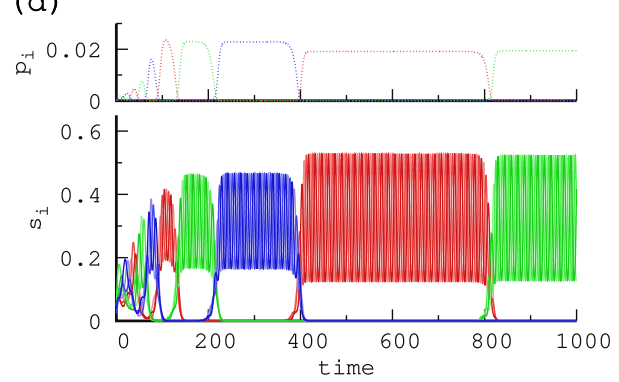

(b)
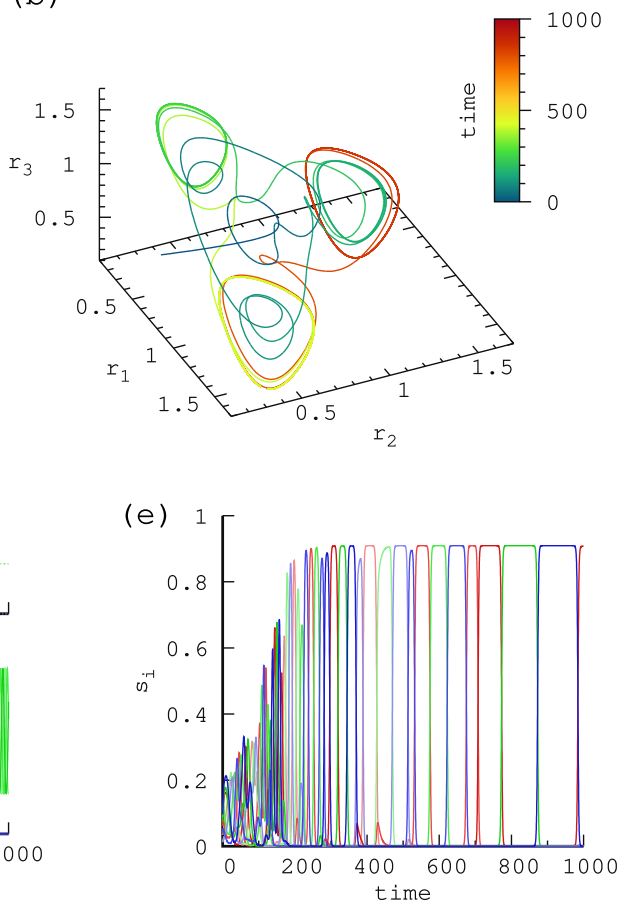

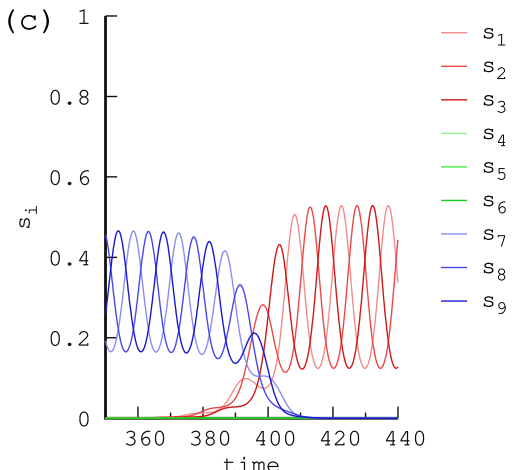

(f)

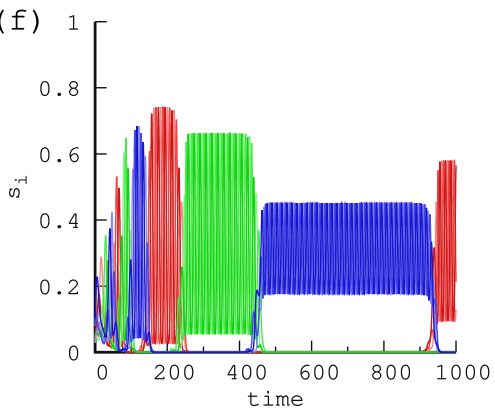

FIG. 5. Dynamics of a heteroclinic cycle between manifolds. (a) Sketch of this motion. Within each of the subspaces $\mathcal{S}_{i}$, the dynamics is conservative and amounts to a periodic orbit. When the subspace/saddle is left in favor of the next saddle, the population densities diminish while those of the subsequent subspace increase. (b) A projection that displaces the subspaces $\mathcal{S}_{i}$ in different coordinates: $r_{1}=s_{1}+s_{2}+s_{3}+$ $\alpha\left(s_{1}+s_{4}+s_{7}\right), r_{2}=s_{4}+s_{5}+s_{6}+\alpha\left(s_{2}+s_{5}+s_{8}\right)$, and $r_{3}=s_{7}+s_{8}+s_{9}+\alpha\left(s_{3}+s_{6}+s_{9}\right)$ with $\alpha=1.5$. (c) Zoom into a time window where a switch from one manifold to another happens. Population densities $s_{i}$ are plotted against time $t$. (d)-(f) Sensitive dependence of the time evolution on the random initial conditions with subsequent switching between manifolds of periodic orbits. Parameters are $\gamma=1.1, r=$ $1.25, e=0.2, f=0.3$, and $c=d=2$. For further explanations, see the text. 
nine-dimensional space. As long as the trajectory remains in the immediate vicinity of the subspace, it is hard to distinguish from a regular periodic orbit.

In this sense, the selected periodic orbit decays after a number of oscillations, since the slow manifold itself is a saddle; the heteroclinic trajectory switches to the next subspace and selects another periodic orbit there. Figure 5(a) shows a sketch of this motion, Fig. 5(b) a projection from nine- to three-dimensional space, where the color codes the time and it is visible that the subspaces are repeatedly visited. In each subspace, the trajectory spirals from an initially almost vanishing concentration toward an almost periodic orbit, remains there for a while, and spirals back to almost zero when the switch to the next subspace occurs. Figure 5(c) corresponds to a zoom into a switch between different subspaces, for which three species $(7,8$, and 9$)$ follow a periodic orbit in the vicinity of $\mathcal{S}_{3}$, indicated by three shades of blue, and a second set, species 1,2 , and 3 (in three shades of red) in the vicinity of the slow manifold within $\mathcal{S}_{1}$.

Figures 5(d)-5(f) show the strong impact of the initial conditions (here chosen randomly). Due to the conserved product, the initial conditions fix the periodic orbit very close to the respective first subspace, and since the dynamics is fully deterministic, all subsequent switching between different subspaces of periodic orbits at later times are determined. The upper plot in Fig. 5(d) shows the time evolution of the products $p_{i}$ with dotted lines. Figures 5(d) and 5(e) clearly differ by the number of periodic orbits performed in the vicinity of the subspace with rapid switching between the subspaces $\mathcal{S}_{i}$ in Fig. 5(e). The amplitude of the periodic orbit does not monotonically change in time if the same plane is revisited later, as seen in Fig. 5(f); only the dwell time of the trajectory spent close to the subspace increases monotonically with time as is typical for a heteroclinic motion.

This way, the setting produces a heteroclinic cycle between three saddles which are manifolds with conservative dynamics inside these manifolds. The heteroclinic cycle displays typical fast transitions between the saddles, interrupted by increasingly longer visits to the saddles, while starting a trajectory precisely within one of the manifolds leads to a stable periodic orbit. An infinity of such orbits is available due to the conserved quantity. Thus, manifolds as identified in Sec. II can establish a heteroclinic cycle if embedded in a higher-dimensional space within which they have saddle-type stability.

\section{B. Critical regime with slow decay of periodic orbits}

Figure 6 illustrates the decay of periodic orbits in the critical regime on the side where the coexistence equilibrium is stable as in Fig. 6(a) and where the heteroclinic cycle is attractive within the subspaces $\mathcal{S}_{i}$ as in Fig. 6(b). Outside the critical regime, the periodic orbits as precursors of the dynamics at the bifurcation point are gone, the trajectories are no longer delayed by long-lasting periodic orbits, and quicly reach the vicinity of their final destination as in the previous section. The scaling in this critical regime of the decay of the conserved product in subspaces $S_{i}$ can be derived as follows. With $\gamma$ denoting the bifurcation parameter and $\gamma=\frac{c+e}{2}$ the bifurcation point, we use $\epsilon$ to parameterize the
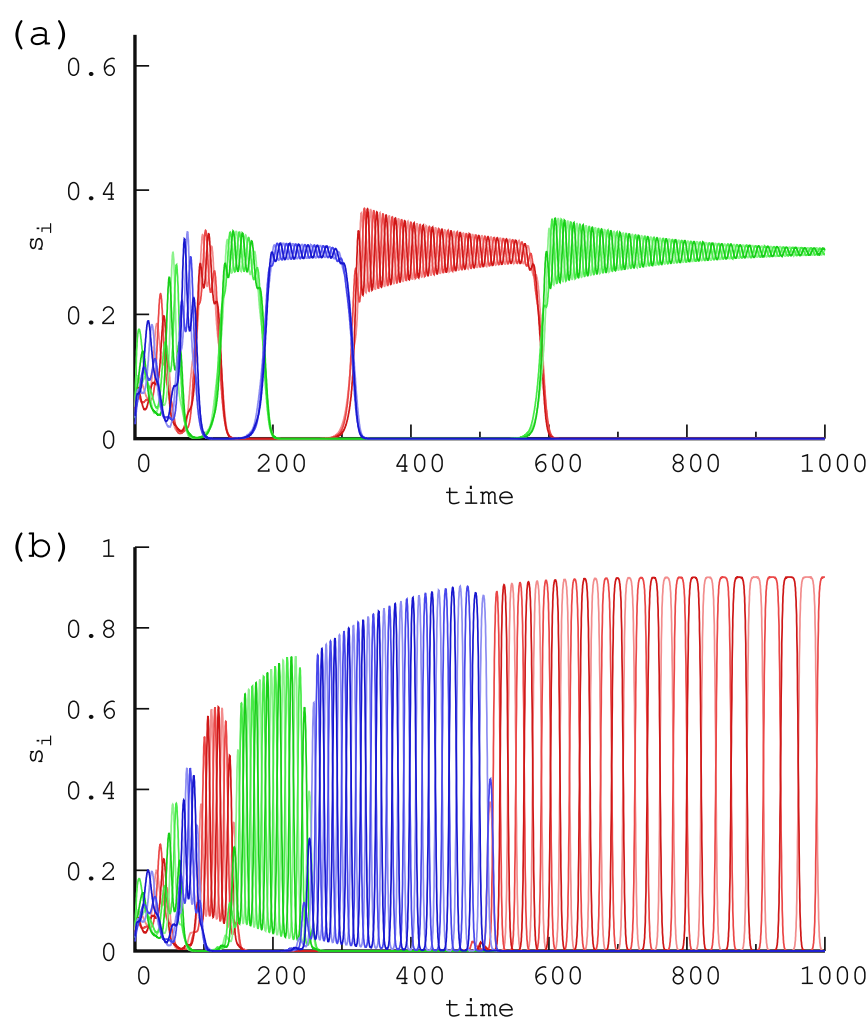

FIG. 6. Dynamics in the critical regime. (a) Slightly above the bifurcation point (here $\gamma=1.12$ ) with a slow decay of the periodic orbits after reaching the subspace $\mathcal{S}_{i}$ it is embedded in. (b) Slightly below the bifurcation point (here $\gamma=1.08$ ) with a slow decay of the periodic orbits. Parameters are $\gamma=1.1, r=1.25, e=0.2, f=0.3$, and $c=d=2$.

distance from the bifurcation point. Using $B$ from Eq. (12), we find $B_{i, i+1}=c-\epsilon-\frac{c+e}{2}=\frac{c-e}{2}-\epsilon$ and $B_{i, i+2}=e-\epsilon-$ $\frac{c+e}{2}=-\frac{c-e}{2}-\epsilon$. Obviously, $\mathbf{B}$ is skew symmetric when $\epsilon=0$. Equation 11 thus becomes

$\mathrm{d}_{t} s_{i}=s_{i}\left(1-\sum_{j} \gamma s_{j}\right)-s_{i} s_{i+1}\left(\frac{c-e}{2}-\epsilon\right)+s_{i} s_{i+2}\left(\frac{c-e}{2}+\epsilon\right)$,

when restricted to $\mathcal{S}_{i}$. Using Eq. (13) in $\mathrm{d}_{t} \prod_{i} s_{i}$, we find

$$
\begin{aligned}
\mathrm{d}_{t} \prod_{i} s_{i} & =\prod_{i} s_{i}\left(3\left(1-\sum_{i}\left(\epsilon+\frac{c+e}{2}\right) s_{i}\right)+2 \epsilon \sum_{i} s_{i}\right) \\
& \approx-\epsilon \prod_{i} s_{i} \cdot \sum_{i} s_{i},
\end{aligned}
$$

where we have used that $\sum_{i} s_{i}=1 /\left(\frac{c+e}{2}\right)$ close to the plane, so $\epsilon$ may be absorbed in a rescaling of time, and the time evolution of the products of species spanning the different subspaces would collapse to a master curve in the critical regime.

\section{COUPLED HETEROCLINIC NETWORKS ON A GRID}

The hierarchical heteroclinic network that we have described so far is a network in phase space. With two hierarchy levels, it is supposed to describe transient heteroclinic 

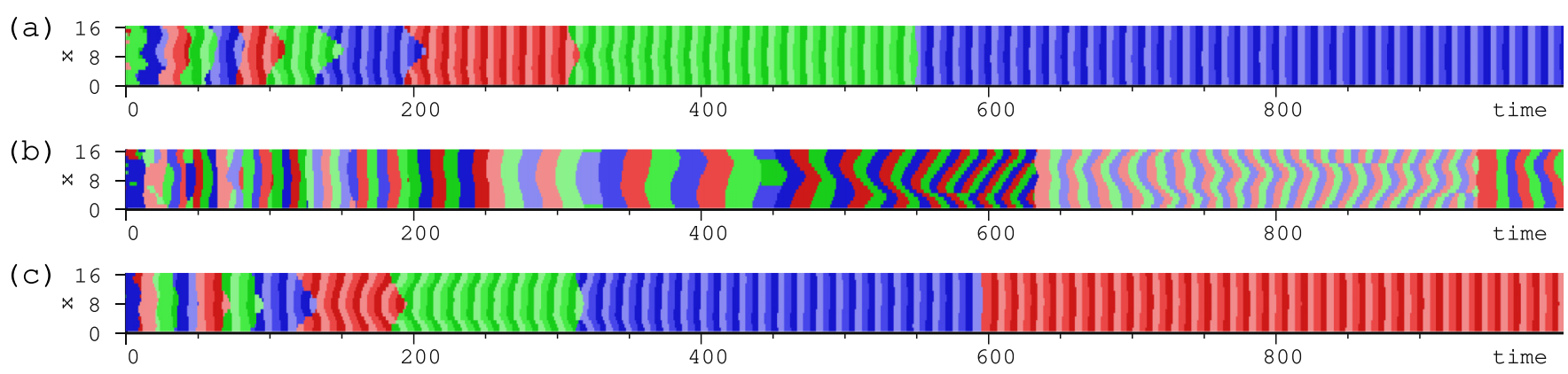

FIG. 7. Strong dependence on the initial conditions at the bifurcation point as a further source of a variety of patterns on spatial grids, here represented by a vertical one-dimensional extension of 16 units. Color codes the dominating species at each point in time $t$ and site in space $x \in\{1 \ldots 16\}$. Panels (a)-(c) use the same initial condition as panels (d)-(f) in Fig. 5, respectively, but add random values (drawn uniformly from $\left.[0,0.05]^{9}\right)$ at all sites for spatial variation. Parameters: $\delta=0.05, \gamma=1.1, r=1.25, e=0.2, f=0.3$, and $c=d=2$.

dynamics with a slow and a fast timescale. On the other hand, brain dynamics seems to be organized in multiple hierarchical processes which are ongoing in parallel [32]. If we couple hierarchical heteroclinic networks on a spatial grid, it provides a possible implementation of this organizational feature that is shared with brain dynamics. Therefore, we studied in Ref. [20] sets of heteroclinic networks with two and three hierarchy levels, diffusively coupled on larger grids of size $128 \times 128$ or $256 \times 256$. Interestingly, for a certain range of parameters, in particular for sufficiently large diffusion constants, we observed a final state that amounts to a large dimensional reduction of the high-dimensional phase space to the space of a single hierarchical network when all units synchronize toward the same heteroclinic trajectory. The final state of full synchronization may be preceded by no or partial synchronization between heteroclinic cycles at different sites which then can lead to nested spiral patterns. The patterns are conclusive of the spatial distribution of the temporal winners of the competition between the species or the information items.

In principle, we expect that the rich dynamical repertoire at individual sites of single heteroclinic networks at the very bifurcation points manifests itself in an increased variety of patterns if the individual units are tuned toward their bifurcation points. Here we restrict the considerations to the final state of a fully synchronized grid of heteroclinic networks with two hierarchy levels. In Fig. 7, we have started with the same initial conditions as in Figs. 5(d)-5(f), respectively, but added random values (drawn uniformly from $[0,0.05]^{9}$ ) at all sites for some initial spatial variation. We plot the three sequences as a function of time, where the color codes the temporarily dominant species and the vertical extension represents a one-dimensional grid of sixteen sites. If the diffusive coupling is chosen sufficiently strong such that the whole grid follows the same periodic orbits, we observe temporal sequences of uniformly colored vertical stripes being fully synchronized. The color changes are fast between different shades of a certain color and take longer between different colors. However, in detail, these sequences, corresponding to the three initial conditions, are quite different in the distribution of time intervals between subsequent flips and in the sequence of colors themselves.

Already, without the proliferation of the dynamical repertoire at the bifurcation points, the patterns are versatile and sensitive to the initial conditions, the diffusion constant, and the choice of other parameters. Here we only point toward a further source of enrichment of the possible patterns.

\section{CONCLUSION}

In various examples of Hopf bifurcations in heteroclinic dynamics, we have seen that posing the system right at a bifurcation point reveals a large dynamical repertoire, where a conserved quantity enables a strong dependence on the initial conditions with new invariant sets emerging at the bifurcation point such as an infinite set of periodic orbits, while heteroclinic cycles or equilibria are observed away from the bifurcation points. The most interesting example was the emergence of an infinite set of cyclic heteroclinic trajectories between manifolds. It happens at the bifurcation point at which two hierarchy levels get reduced to a single one in a hierarchical heteroclinic network. The infinite set is generated by the set of initial conditions. Depending on the initial condition, each heteroclinic trajectory temporarily selects a certain periodic orbit from an infinite set of periodic orbits which densely cover each of the three manifolds. These manifolds are approached in a heteroclinic trajectory during iterated revolutions. The resulting trajectory visits periodic orbits of different amplitudes. It looks like a heteroclinic sequence between periodic orbits, but actually it corresponds to a heteroclinic sequence between manifolds. Each of these manifolds as a whole plays the role of a saddle of the heteroclinic cycle. In this sense, we identified a heteroclinic cycle between manifolds of conservative dynamics. In a small parameter range aside the bifurcation point, precursors of the proliferation of states can be seen, whose lifetime increases with decreasing distance to the bifurcation point. Along with that, quantities which are conserved at the bifurcation points decay slowly in the critical regime.

In this sense, we observe features of dynamical criticality (in contrast to statistical one). It is, however, not self-organized like the self-regulated dynamical criticality, observed in human ECoG (electrocorticography) data [33]. Thus it is open whether at all-and if so-how cognitive dynamics may tune parameters toward these bifurcation points or at least to their vicinity to utilize the rich dynamical repertoire for storage and processing of information. 
However, from a more abstract point of view, our findings illustrate the emergence of different and novel phenomena at the borderline between dynamical regimes. In Ref. [34], the importance of being borderline has been emphasized in relation to the reduction from an encompassing theory to a less general theory that is achieved in some limit that is often singular. An example is the reduction of statistical mechanics to thermodynamics in the thermodynamic limit. According to Ref. [34], singularities are directly connected to the existence of emergent phenomena: "Emergent phenomena inhabit the borderland between theories" [34]. In this paper, we have identified emergent dynamics at the border between different dynamical regimes, separated by degenerate Hopf bifurcations which shared features of critical points: A high sensitivity, here to the initial conditions, with a proliferation of the dynamical repertoire, inherent slow timescales in the vicinity of these points, characterized by slow manifolds, long transients, and slow decay of conserved quantities. A small parameter interval around the bifurcation points shows the typical precursors of the dynamics emerging at the bifurcation points.

In view of heteroclinic dynamics, it will be interesting to see whether the kind of dynamical criticality observed in this paper can actually be exploited when heteroclinic dynamics is used for computation in artificial systems. More generally, it seems challenging to further explore the dynamics "on top" of a whole variety of bifurcation points that are expected if the dynamics is realized as coupled heteroclinic networks on spatial grids, working in parallel at different sites and hierarchically at individual sites.

\section{ACKNOWLEDGMENTS}

We thank the German Research Foundation (DFG Contract No. ME-1332/28-1) for financial support.
[1] T. Mora and W. Bialek, Are biological systems poised at criticality? J. Stat. Phys. 144, 268 (2011).

[2] M. A. Muñoz, Colloquium: Criticality and dynamical scaling in living systems, Rev. Mod. Phys. 90, 031001 (2018).

[3] C. Langton, Computation at the edge of chaos: Phase transitions and emergent computation, Physica D 42, 12 (1990).

[4] H. Yang, W. L. Shew, R. Roy, and D. Plenz, Maximal variability of phase synchrony in cortical networks with neuronal avalanches, J. Neurosci. 32, 1061 (2012).

[5] G. Deco, A. Ponce-Alvarez, P. Hagmann, G. L. Romani, D. Mantini, and M. Corbetta, How local excitation-inhibition ratio impacts the whole brain dynamics, J. Neurosci. 34, 7886 (2014).

[6] A. Palmigiano, T. Geisel, F. Wolf, and D. Battaglia, Flexible information routing by transient synchrony, Nat. Neurosci. 20, 1014 (2017).

[7] T. Duke and F. Jülicher, Active Traveling Wave in the Cochlea, Phys. Rev. Lett. 90, 158101 (2003).

[8] M. O. Magnasco, A Wave Traveling over a Hopf Instability Shapes the Cochlear Tuning Curve, Phys. Rev. Lett. 90, 058101 (2003).

[9] R. Stoop and F. Gomez, Auditory Power-Law Activation Avalanches Exhibit a Fundamental Computational Ground State, Phys. Rev. Lett. 117, 038102 (2016).

[10] G. Deco, M. L. Kringelbach, V. K. Jirsa, and P. Ritter, The dynamics of resting fluctuations in the brain: Metastability and its dynamical cortical core, Sci. Rep. 7, 3095 (2017).

[11] C. Haldeman and J. M. Beggs, Critical Branching Captures Activity in Living Neural Networks and Maximizes the Number of Metastable States, Phys. Rev. Lett. 94, 058101 (2005).

[12] G. Deco and V. K. Jirsa, Ongoing cortical activity at rest: Criticality, multistability, and ghost attractors, J. Neurosci. 32, 3366 (2012).

[13] E. Tognoli and J. A. S. Kelso, The metastable brain, Neuron. 81, 35 (2014).

[14] M. I. Rabinovich, R. Huerta, P. Varona, and V. S. Afraimovich, Transient cognitive dynamics, metastability, and decision making, PLoS Comput. Biol. 4, e1000072 (2008).
[15] M. I. Rabinovich, P. Varona, A. I. Selverston, and H. D. I. Abarbanel, Dynamical principles in neuroscience, Rev. Mod. Phys. 78, 1213 (2006).

[16] V. Afraimovich, I. Tristan, R. Huerta, and M. I. Rabinovich, Winnerless competition principle and prediction of the transient dynamics in a Lotka-Volterra model, Chaos 18, 043103 (2008).

[17] V. S. Afraimovich, M. I. Rabinovich, and P. Varona, Heteroclinic contours in neural ensembles and the winnerless competition principle, Int. J. Bifurc Chaos. 14, 1195 (2004).

[18] M. I. Rabinovich, V. S. Afraimovich, and P. Varona, Heteroclinic binding, Dyn. Syst. 25, 433 (2010).

[19] M. I. Rabinovich, P. Varona, I. Tristan, and V. S. Afraimovich, Chunking dynamics: Heteroclinics in mind, Front. Comput. Neurosci. 8(22), 1 (2014).

[20] M. Voit and H. Meyer-Ortmanns, Dynamics of nested, selfsimilar winnerless competition in time and space, Phys. Rev. Research 1, 023008 (2019).

[21] M. Voit, S. Veneziale, and H. Meyer-Ortmanns, Coupled heteroclinic networks in disguise, Chaos 30, 083113 (2020).

[22] R. M. May and W. J. Leonard, Nonlinear aspects of competition between three species, SIAM J. Appl. Math. 29, 243 (1975).

[23] F. H. Busse and K. E. Heikes, Convection in a rotating layer: A simple case of turbulence, Science 208, 173 (1980).

[24] J. Guckenheimer and P. Holmes, Structurally stable heteroclinic cycles, Math. Proc. Cambridge Philos. Soc. 103, 189 (1988).

[25] J. Hofbauer and K. Sigmund, Evolutionary Games and Population Dynamics (Cambridge University Press, Cambridge, UK, 1998).

[26] J. Hofbauer, Evolutionary dynamics for bimatrix games: A hamiltonian system? J. Math. Biology. 34, 675 (1996).

[27] J. Knebel, T. Krüger, M. F. Weber, and E. Frey, Coexistence and Survival in Conservative Lotka-Volterra Networks, Phys. Rev. Lett. 110, 168106 (2013).

[28] H. Y. Shih, T. L. Hsieh, and N. Goldenfeld, Ecological collapse and the emergence of travelling waves at the onset of shear turbulence, Nat. Phys. 12, 245 (2015). 
[29] M. Voit and H. Meyer-Ortmanns, A hierarchical heteroclinic network: Controlling the time evolution along its paths, Eur. Phys. J. Special Topics 227, 1101 (2018).

[30] M. Voit and H. Meyer-Ortmanns, Predicting the separation of time scales in a heteroclinic network, Appl. Math. Nonlinear Sci. 4(2), 279 (2019).

[31] P. Ashwin and C. Postlethwaite, On designing heteroclinic networks from graphs, Physica D: Nonlinear Phenom. 265, 26 (2013).
[32] S. Grossberg, The complementary brain: Unifying brain dynamics and modularity, Trends Cognit. Sci. 4, 233 (2000).

[33] G. Solovey, K. J. Miller, J. Ojemann, M. O. Magnasco, and G. A. Cecchi, Self-regulated dynamical criticality in human ECoG, Frontiers Integrative Neurosci. 6, 44 (2012).

[34] M. Berry, Asymptotics, Singularities and the Reduction of Theories, in Logic, Methodology and Philosophy of Science IX, Studies in Logic and the Foundations of Mathematics, Vol. 134, edited by D. Prawitz, B. Skyrms, and D. Westerståhl (Elsevier Science B.V., Amsterdam, 1995), pp. 597-607. 